\title{
BMJ Global Health Epidemiology of multimorbidity in conditions of extreme poverty: a population-based study of older adults in rural Burkina Faso
}

\author{
Maria Lisa Odland (D) , ${ }^{1}$ Collin Payne, ${ }^{2}$ Miles D Witham, ${ }^{3,4}$ Mark J Siedner, ${ }^{5,6}$ \\ Till Bärnighausen, ${ }^{6,7}$ Mamadou Bountogo, ${ }^{8}$ Boubacar Coulibaly, ${ }^{8}$ \\ Pascal Geldsetzer, ${ }^{9}$ Guy Harling (1) , 6,10,11,12 Jennifer Manne-Goehler, ${ }^{13}$ \\ Lucienne Ouermi, ${ }^{8}$ Ali Sie, ${ }^{8}$ Justine I Davies ${ }^{1,11}$
}

To cite: Odland ML, Payne C, Witham MD, et al. Epidemiology of multimorbidity in conditions of extreme poverty: a population-based study of older adults in rural Burkina Faso. BMJ Global Health 2020;5:e002096. doi:10.1136/ bmjgh-2019-002096

Handling editor Sanni Yaya

- Additional material is published online only. To view please visit the journal online (http://dx.doi.org/10.1136/ bmjgh-2019-002096).

Received 18 0ctober 2019 Revised 11 February 2020 Accepted 15 February 2020

Check for updates

(C) Author(s) (or their employer(s)) 2020. Re-use permitted under CC BY-NC. No commercial re-use. See rights and permissions. Published by BMJ.

For numbered affiliations see end of article.

Correspondence to

Maria Lisa Odland;

m.l.odland@bham.ac.uk

\section{ABSTRACT}

Introduction Multimorbidity is a health issue of increasing importance worldwide, and is likely to become particularly problematic in low-income countries (LICS) as they undergo economic, demographic and epidemiological transitions. Knowledge of the burden and consequences of multimorbidity in LICs is needed to inform appropriate interventions.

Methods A cross-sectional household survey collected data on morbidities and frailty, disability, quality of life and physical performance on individuals aged over 40 years of age living in the Nouna Health and Demographic Surveillance System area in northwestern Burkina Faso. We defined multimorbidity as the occurrence of two or more conditions, and evaluated the prevalence of and whether this was concordant (conditions in the same morbidity domain of communicable, non-communicable diseases (NCDs) or mental health (MH)) or discordant (conditions in different morbidity domains) multimorbidity. Finally, we fitted multivariable regression models to determine associated factors and consequences of multimorbidity.

Results Multimorbidity was present in 22.8 (95\% $\mathrm{Cl}, 21.4$ to 24.2) of the study population; it was more common in females, those who are older, single, more educated, and wealthier. We found a similar prevalence of discordant $11.1(95 \% \mathrm{Cl}, 10.1$ to 12.2$)$ and concordant multimorbidity $11.7(95 \% \mathrm{Cl}, 10.6$ to 12.8$)$. After controlling for age, sex, marital status, education, and wealth, an increasing number of conditions was strongly associated with frailty, disability, low quality of life, and poor physical performance. We found no difference in the association between concordant and discordant multimorbidity and outcomes, however people who were multimorbid with NCDs alone had better outcomes than those with multimorbidity with NCDs and MH disorders or MH multimorbidity alone.

Conclusions Multimorbidity is prevalent in this poor, rural population and is associated with markers of decreased physical performance and quality of life. Preventative and management interventions are needed to ensure that health systems can deal with increasing multimorbidity and its downstream consequences.

\section{Key questions}

What is already known?

- Multimorbidity is an increasing health problem worldwide, but the burden in many low income, and particularly the least developed, countries remains unknown.

- How multimorbidity relates to functional outcomes and quality of life in people living in these regions has not been well documented; there is very little evidence from least developed countries.

What are the new findings?

- In Burkina Faso, one of the poorest countries in the world, multimorbidity, defined as two or more conditions, is present in over $20 \%$ of those aged over 40 in a rural population.

- Multimorbidity prevalence increases with age, is more common in women, in those who have more education, are unmarried or are in the highest relative economic category.

- Increasing numbers of morbidities are associated with frailty, disability, lower quality of life and poore physical performance.

- People who are multimorbid with non-communicable diseases (NCDs) alone have better outcomes than those who those who have concurrent NCDs and mental health conditions or are multimorbid with mental health conditions alone.

What do the new findings imply?

- Multimorbidity is a global health issue of major significance requiring substantial investment in health system funding to combat.

- Adopting a more holistic care provision strategy, instead of siloed care, could provide an avenue towards better health.

\section{INTRODUCTION}

Over the past decades, there has been substantial progress in reducing infectious diseaserelated morbidity and improving maternal 
and child health. ${ }^{1-3}$ These successful efforts, combined with rapid economic development, have resulted in an ageing population in low-income and middle-income countries (LMICs), and especially in ub-Saharan Africa. ${ }^{45}$ New disease and mortality patterns have accompanied these transitions and non-communicable diseases (NCD) and mental health (MH) disorders have emerged as important issues. ${ }^{6-8}$ A change in development focus has paralleled this epidemiological shift with the sustainable development goals moving away from single diseases, to a broader array of conditions and access to the health systems required to treat these. ${ }^{9}$ These changes have come with a realisation that individuals often suffer from multiple concurrent conditions, and that the circumstances in which individuals live impacts both risk of conditions and their physical consequences. ${ }^{1011}$

Although there have been studies on multimorbidity in lower-middle income countries most of them have been done in upper-middle income countries. In sub-Saharan Africa, these have mainly been conducted in South Africa, ${ }^{12}{ }^{13}$ which is an upper-middle-income country with a relatively well-developed health system. ${ }^{14}$ There is very little information about who is affected by multimorbidity in other countries in sub-Saharan Africa, where many of the least developed countries with the world's poorest populations and most under-resourced health systems are found.

Similarly, there are few data on associations between multimorbidity prevalence and patterns and related health outcomes in the least developed countries, although studies have shown associations with hospitalisations, mortality, disability, low quality of life and poor physical performance in other, more developed countries, countries. ${ }^{15-24}$ Given the differences in context, including wealth, culture and health service availability, it cannot be assumed that relationships between multimorbidity and outcomes found in upper or upper-middle income countries apply to lower income countries (LICs). The increase in the triple burdens of communicable, NCD and MH in LICs means such information is essential to inform investment in preventative and management initiatives, and to shape the development of health systems in these least developed countries. ${ }^{25}$

Importantly, a meaningful definition of multimorbidity that aligns the concept with patient outcomes, rather than based solely on expert opinion, has also not been agreed on. ${ }^{6} 2022$ 26-28 The WHO defines multimorbidity as the coexistence of two or more chronic conditions. ${ }^{29}$ However, it has also been described as the count of conditions, two or more conditions in the disease domains of communicable, non-communicable or $\mathrm{MH}$, and has been further subdivided into discordant (more than two conditions in different domains) or concordant (more than two conditions in the same domain) multimorbidity. ${ }^{6}{ }^{13}$ Showing the relationships between multimorbidity using these definitions and functional outcomes in one of the poorest countries in the world is useful to policy makers, funders and researchers.
Burkina Faso is a small landlocked LIC in sub-Saharan Africa with limited natural resources; it is ranked 183 of 189 countries on the Human Development Index. ${ }^{30}$ It has a population of about 19 million people which is growing rapidly. ${ }^{30}{ }^{31}$ There are few data on the epidemiology of multimorbidity in Burkina Faso, although one study suggested a high prevalence of multimorbidity in an urban setting. ${ }^{32}$ This study aims to investigate the prevalence and associations (including important downstream consequences of multimorbidity including frailty, disability, quality of life and other measures of physical performance) among middle-aged and older individuals in a rural, very low income setting in Burkina Faso. We sought to assess the prevalence of multimorbidity, both with concordant conditions (defined as two or more conditions in the same morbidity domain of communicable, NCD or MH) and discordant conditions (defined as two or more conditions in different morbidity domains) in rural Burkina Faso, explore how multimorbidity varies by sociodemographic characteristics in this population and to assess whether it is correlated with related outcomes such as frailty, disability, low quality of life and poor physical performance.

\section{METHODS}

\section{Study setting}

The study was set in the Nouna Health and Demographic Surveillance System (HDSS) area, led by the Centre de Recherche en Santé de Nouna (CRSN). The HDSS collects data, yearly, on births, deaths and migration in a well-enumerated population in the Boucle du Mouhoun region, north-western Burkina Faso. The demographic surveillance area of the Nouna HDSS consist of the market town of Nouna and 59 surrounding villages with a total population of $107000 .{ }^{33}$ Residents come from multiple ethnic groups (Bwaba, Dafin, Mossi, Peulh and Samo) and have a range of religious beliefs (Animist, Muslim, Protestant and Roman Catholic). The major economic activities are farming and animal husbandry.

\section{Sample}

Data for this cross-sectional study were obtained during the baseline wave of the CRSN Heidelberg Aging Study (CHAS). CHAS consists of a population-representative sample of adults $\geq 40$ years of age living in the Nouna HDSS area. To obtain a sample of 3000 older adults, we used a two-part multistage random sample of 4000 individuals from the 2015 Nouna HDSS census, allowing for $25 \%$ loss due to mortality, mobility or non-response. The sample size of 3000 was chosen to detect meaningful differences in the prevalence of hypertension and diabetes across key demographic and lifestyle factors. In all villages with fewer than 90 adults aged over 40 , all adults were selected to take part. In all other villages, a random sample of households with at least one person over 40 years old was drawn, and then within each selected household one 
age-eligible adult was randomly selected to complete the survey.

\section{Data collection}

Data collection was performed between May and July 2018. Data were collected using Open Data Kit software on tablet computers at the participants' residence. ${ }^{34}$ Interviews were conducted in either French or translated by the interviews into the local languages of Dioula or Mooré. The household survey questionnaire contained questions on age; gender; education; marital status; household assets and self-reported medical conditions. Cognition was assessed using the eight question community screening interview for dementia (CSI-D), ${ }^{35}$ anxiety using the Generalised Anxiety Disorder question (GAD-2) score $^{36}$ and depression using Patient Health Questionnaire (PHQ-9). ${ }^{37}{ }^{38}$ Questions on weight loss in the past year, self-reported activity and levels of exhaustion were used in construction of the Fried frailty score. ${ }^{39} 40$

Height and weight were measured using generic scales and measurement bands against a vertical and horizontal smooth surface, respectively. Blood pressure was measured in a seated position in the left arm after $15 \mathrm{~min}$ rest; three measures were taken using Omron Series 7 portable blood pressure machines (Omron Healthcare, Kyoto, Japan) with the second and third measurements taken at least $5 \mathrm{~min}$ apart. The mean of the second and third measurements was used in analyses. Capillary glucose levels were analysed using SD Biosure Codefree point-of-care testing strips, and cholesterol using the Jactron Pictus 400 machine. Three measures of physical performance were obtained. Chair rise was measured by recording the time taken to make five rises from a standardised collapsible armless chair using a digital stopwatch; participants were asked to stand without using their arms to assist. ${ }^{41}$ Walk speed was measured over a 4-m course marked out on level ground. ${ }^{41}$ Participants were asked to walk their regular pace from a standing start. Two measures were taken, doing the course in each direction, and time was taken using a digital stopwatch and recorded to the nearest tenth of a second. The fastest speed in metres per second $(\mathrm{m} / \mathrm{s})$ was used. Hand grip strength was measured using a Jamar Hydraulic Hand Dynamometer. ${ }^{42}$ Measurements were taken with the participant seated, the arm at $90^{\circ}$ elbow flexion and the shoulder and wrist in the neutral position. Two attempts were recorded from each hand; the maximum was used in this analysis.

\section{Definition of variables}

\section{Demographic characteristics}

Marital status was categorised as married/cohabiting or single/widowed/divorced. Educational level was categorised as no education or any education. Participants were asked 37 questions on household assets and dwelling characteristics; from these, wealth quintiles were derived from the first principal component using the method of Filmer and Pritchett. ${ }^{43}$

\section{Definitions of disease states}

A prior diagnosis of cancer, HIV, chronic respiratory disease, stroke and heart disease relied on self-report of diagnosis (eg, 'Have you ever been told by a health worker that you have cancer?'). Hypertension was defined as either self-reported previous diagnosis or currently being on treatment, or as a measured systolic blood pressure $\geq 140 \mathrm{~mm} / \mathrm{Hg}$, or a diastolic blood pressure $\geq 90 \mathrm{~mm}$ / Hg. Diabetes was defined as either self-reported diagnosis; being on treatment; a non-fasting point of care capillary glucose level $>200 \mathrm{mg} / \mathrm{dL}, \mathrm{HbAlc}>6.5 \%$ or fasting glucose $>126 \mathrm{mg} / \mathrm{dL}$. Hypercholesterolaemia was defined as a total plasma cholesterol $>200 \mathrm{mg} / \mathrm{dL}$, low density lipoprotein $>160 \mathrm{mg} / \mathrm{dL}$, or self-reported diagnosis or treatment.

Participants were defined as having symptoms of anxiety based on a GAD-2 score $\geq 3 .{ }^{36}$ Participants scoring $0-4$ on PHQ-9 were defined as no depressive symptoms, those scoring 5-9 mild depressive symptoms, 10-14 moderate depressive symptoms, 15-19 moderate-severe depressive symptoms and $\geq 20$ severe depressive symptoms; we categorised participants with moderate or greater symptoms as having depressive symptoms in this analysis. ${ }^{38}$ Cognitive functioning was measured using Community Screeing Instrument for Dementia (CSI-D) with a maximum score of nine, and divided into normal or possible/probable cognitive impairment if a score under seven. ${ }^{44}$

The Fried score was used to calculate frailty using the original five domains (weight loss, low grip strength, walk speed, activity levels and exhaustion). We chose to use the Fried score to calculate frailty as it can easily be deployed in a low resource setting, given that it uses just five parameters. ${ }^{12}{ }^{39}$ The Fried score is a validated method which is shown to be feasible and comparable to other methods for calculating frailty. ${ }^{45}$ Cut-offs for each domain of frailty were selected to be as close to the original Fried frailty score as possible (detailed definitions published elsewhere) ${ }^{39}$ Weight loss was defined as self-reported loss of $>4 \mathrm{~kg}$ over the last 12 months, low grip strength as the lowest quintile of BMI-adjusted grip for each sex and low walk speed as the lowest quintile of height-adjusted walk speed over a 4-m course for each sex. Low activity levels were defined as the highest quintile of self-reported hours of sitting per week for each sex. Self-reported exhaustion was measured using two questions from the Center for Epidemiologic Studies Depression Scale (CES-D) scale: 'Everything I did in the last week was an effort' or 'I could not get going,. ${ }^{47}$ A positive response was defined as either statement applying for at least 3-4 days per week.

Each domain (low grip strength, low walk speed, or self-reported weight loss, exhaustion or low activity levels) scored 1 point giving a final total between 0 and 5 points. Individuals were categorised as non-frail/robust (0 points), prefrail (1-2 points) or frail (3-5 points). Participants with missing data in one or more domains were labelled as 'as unable to calculate'; previous work in South Africa has suggested that such individuals have a 
health prognosis similar to or worse than those classified as frail, therefore they were included in frail category. ${ }^{46}$

Disability was measured using the 12-item WHO Disability Assessment Schedule, version 2 (WHODAS V.2.0) disability score. ${ }^{114}$ This score measures impairments in function, activity and participation (mobility, self-care, cognition, interaction with others, life activities and social participation). Measures were summed to form a score, then normalised to a 0-100 scale, where 0 equals no disability and 100 represents the worst disability. Quality of life was measured using the validated EuroHIS 8-item version of the WHO Quality of Life (WHOQOL). ${ }^{11} 49$ Each item was scored on a fivepoint scale and summed: for the analysis, the score was normalised to a $0-100$ scale, with 100 denoting the best quality of life.

\section{Defining multimorbidity}

First, count of morbidities (regardless of domain) was described. Then, morbidities were categorised into seven groups within three domains as follows: Noncommunicable: Cardiovascular (hypertension, diabetes, hypercholesterolaemia, heart disease or stroke), chronic respiratory disease (chronic pulmonary obstructive disease or asthma) or cancer. Communicable: prior HIV. Mental Health: cognitive impairment, or symptoms of anxiety or symptoms of depression. Multimorbidity was defined as presence of two or more conditions (regardless of domain); two or more conditions, with at least one in a different domain (discordant multimorbidity); or two or more conditions that are all in the same domain (concordant multimorbidity). Given multimorbidity with NCDs alone has been associated with better outcomes than with NCDs and $\mathrm{MH}$, a separate analysis was done looking at associations of multimorbidity with NCDs, MH or both with functional outcomes. ${ }^{60}$ Since some of the conditions are self-reported, a separate sensitivity analysis was done using only objective measurements which included six conditions (hypertension, diabetes, hypercholesterolemia, cognitive impairment, symptoms of anxiety or symptoms of depression).

\section{Patient and public involvement statement}

Participants were not directly involved in planning the study as it was done as a part of regular demographic health survey. Information was regularly fed back to the participants as by protocol for a demographic health survey.

\section{Statistical analysis}

All analyses were done using SPSS V.24. Variables were described using mean and SD, or median and IQR if not normally distributed. Categorical variables were described using count and proportion. Only participants with data on all the outcome variables were included in the analysis. Bivariate associations between individual morbidities or morbidity categories and functioning outcomes were tested using the $\chi^{2}$ test for categorical variables and student's t-test or Mann-Whitney-U for normally or non-normally distributed continuous variables, respectively. Multivariable models were fit using general linear modelling using a gamma distribution and a log link for skewed dependent variables. We present OR and $95 \%$ CIs, estimated marginal means (EM) and beta values with standards errors, where appropriate. Inverse probability of response weights for survey participation were calculated using gender-specific logistic models for study participation given sample selection. The model included age category, religion, ethnicity and village, and we used these weights in all analyses. Of note, $95.3 \%$ of households supplied only one person to take part in the survey; we therefore assumed clustering at the household level to be minimal and did not adjust for it.

\section{RESULTS}

Of the 3998 sampled individuals, 3033 agreed to participate and completed a study questionnaire. Only participants with information on all conditions were included leaving us with a sample of $2604(85.9 \%)$ people in our analysis. Missing data on HIV status was the most common reason for exclusion $(n=218)$. There was no significant difference between the included individuals and those that were excluded due to missing values (online supplementary appendix table 1). Weighted proportions for all variables used in the study are presented in table 1 . Notably, $50.1 \%$ of the study population were women, a most participants were aged between 40 and 49 (41.7\%), $92.5 \%$ had no formal education and $74.9 \%$ were married or cohabiting; $48.8 \%$ (95\% CI 16.7 to 34.2 ) of the population had at least one NCD, with hypertension being the most prevalent $(36.7 \%, 35.2$ to 38.4$)$, while $0.6 \%(0.04$ to 0.09$)$ had a communicable disease, and $21.1 \%(77.6$ to 80.3) had symptoms suggestive of any MH. Approximately half $(47.8 \%, 46.1$ to 49.6$)$ of the population was prefrail and $8.0 \%$ (7.1 to 9.0 ) were frail, the median disability score was 8.3 (IQR 0.0-22.9) and the mean quality of life score was 59.97 (SD 14.4). Almost one-quarter 22.8 (21.4 to 24.2) of respondents were multimorbidity using the liberal definition of two or more conditions, where half of them had concordant $(11.7 \%, 10.6$ to 12.8$)$ and half had discordant $(11.1 \%, 10.1$ to 12.2$)$ multimorbidity; 9.3\% (8.4 to 10.4 ) had multimorbidity with only NCDs, while $2.5 \%$ (2.04 to 3.09) had multimorbidity with only MH and $10.9 \%$ (9.9 to 12.0 ) had multimorbidity with both MH and NCDs.

Multivariable associations between population characteristics; morbidity domain of NCD, communicable disease or $\mathrm{MH}$; or multimorbidity category of two or more conditions, discordant or concordant are presented in table 2 (bivariate associations are shown in online supplementary appendix table 2). Associations with increasing numbers of conditions are also shown. Female sex; increasing age; increasing education level; or increasing wealth; or being widowed, single or divorced were associated with a greater number of conditions, 
Table 1 Weighted proportions of demographic characteristics and outcomes of included study participants ( $\mathrm{n}=2604)$

\begin{tabular}{|c|c|c|}
\hline Parameter & Group & $\%$ of population unless stated \\
\hline Female & & 50.1 \\
\hline \multirow[t]{3}{*}{ Age } & $40-49$ & 41.7 \\
\hline & $60-69$ & 18 \\
\hline & $70-79$ & 10.2 \\
\hline Education level & Some education & 7.5 \\
\hline \multirow[t]{2}{*}{ Marital status } & Widowed/divorced/single & 25.1 \\
\hline & Married/cohabiting & 74.9 \\
\hline \multirow[t]{2}{*}{ Wealth quintile (5 is the most wealthy) } & 1 & 19.5 \\
\hline & 5 & 20.7 \\
\hline \multirow[t]{8}{*}{ Non-communicable diseases } & Hypertension & 36.7 \\
\hline & Diabetes & 6.7 \\
\hline & Hypercholesterolaemia & 10.9 \\
\hline & Heart disease & 5.7 \\
\hline & Stroke & 1.2 \\
\hline & Chronic respiratory disease & 3.2 \\
\hline & Cancer & 0.5 \\
\hline & Proportion of people with at least 1 NCD & 48.8 \\
\hline \multirow[t]{3}{*}{ Frailty } & Not frail & 44.2 \\
\hline & Prefrail & 47.8 \\
\hline & Frail & 8.0 \\
\hline Disability & WHO DAS score (0-100) median (IQR) & $8.33(0-22.92)$ \\
\hline Quality of life & WHO QoL score (0-100) mean (SD) & $59.97(14.43)$ \\
\hline \multirow[t]{5}{*}{ Physical functioning } & Chair rise time (s) median (IQR) & $14.00(11.99-17.00)$ \\
\hline & (179 missing) & \\
\hline & 4-m walk speed (m/s) mean (SD) & $0.96(0.26)$ \\
\hline & (32 missing) & \\
\hline & Grip strength mean (SD) & $37.88(11.32)$ \\
\hline \multirow[t]{3}{*}{ Multimorbidity categorisation } & Count $>2$ & 22.8 \\
\hline & Count $>3$ & 8.0 \\
\hline & Count $>4$ & 2.4 \\
\hline \multirow[t]{2}{*}{ Multimorbidity categorisation } & $\begin{array}{l}\text { Concordant (>2 conditions with each in the same } \\
\text { category) }\end{array}$ & 11.7 \\
\hline & $\begin{array}{l}\text { Discordant (>2 conditions with each in different } \\
\text { categories) }\end{array}$ & 11.1 \\
\hline
\end{tabular}

CD, communicable disease; DAS, Disability Assessment Schedule; MH, mental health; NCD, non-communicable disease; QoL, quality of life. 


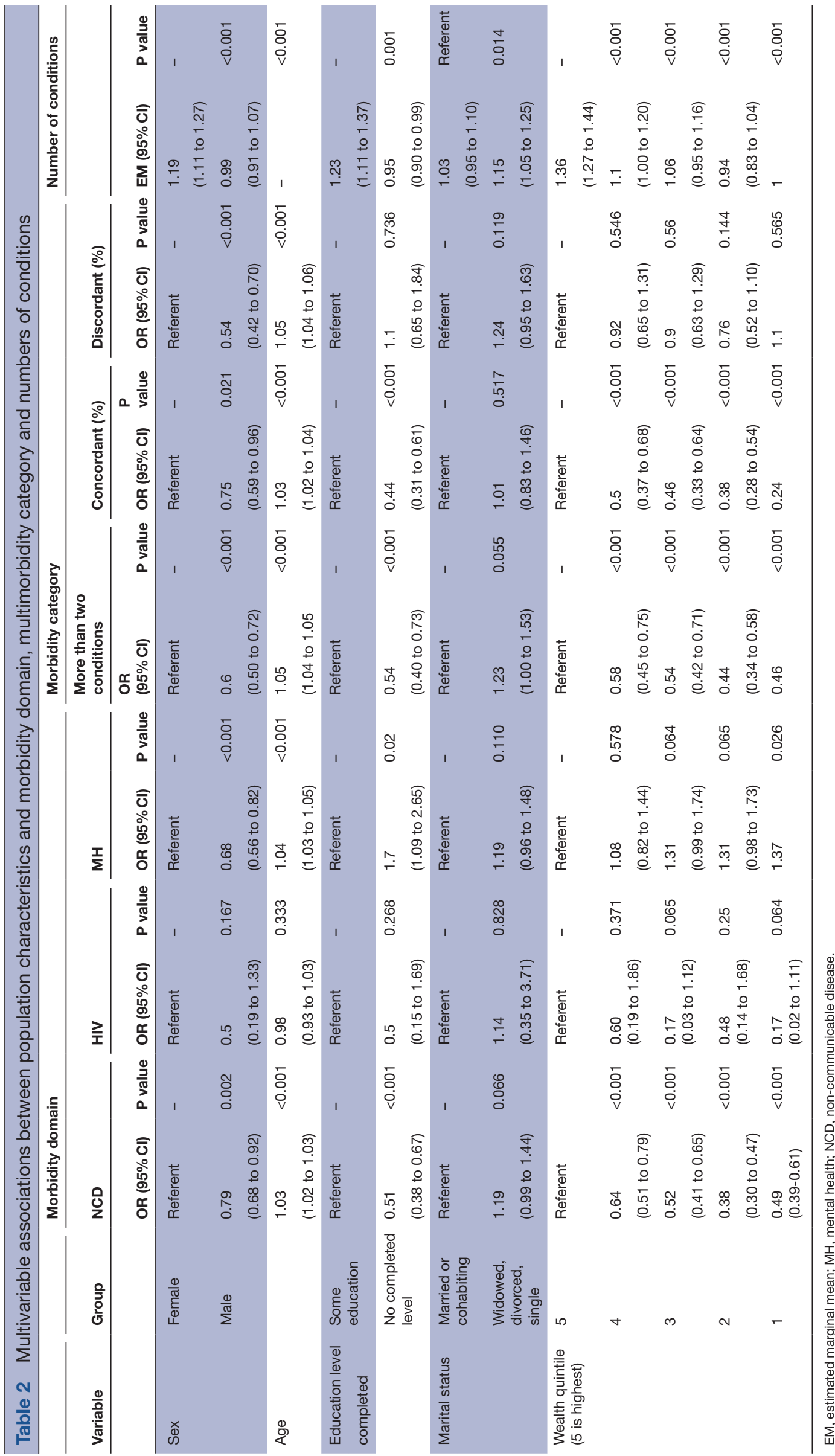


and all of them except marital status were also associated with being multimorbid ( $>2$ conditions). Women were more likely than men to have a condition in the domains of NCD or $\mathrm{MH}$, but there was no association between sex and communicable diseases. Women were also more likely than men to be multimorbid whether concordant or discordant. Increasing age was associated with increased likelihood of having morbidities in the NCD or MH domains, or being multimorbid, whether concordant or discordant. Longer length of education was associated with a greater likelihood of having NCDs, and greater likelihood of concordant multimorbidity. On the other hand, people with some education were less likely of having MH symptoms. Higher wealth was positively associated with NCDs and concordant multimorbidity, but negatively associated with $\mathrm{MH}$ symptoms. In the sensitivity analysis only using objective measurements female gender remained associated NCDs, $\mathrm{MH}$, increased number of conditions and having concordant or discordant multimorbidity. Age continued to be positively associated with having a NCD, MH, increased number of conditions and multimorbidity (concordant or discordant). Similarly, education was associated with higher likelihood of NCDs, but lower risk of $\mathrm{MH}$, and an all over higher risk of having concordant multimorbidity (online supplementary appendix table 3 ). Wealth was associated with NCDs and concordant multimorbidity, but only the lowest wealth quintile had a significantly increased risk of having MH (online supplementary appendix table 3 ). Associations between individual characteristics and being multimorbid in the domains of NCDs and MH can be seen in online supplementary appendix table 4 . Women were more likely to have multimorbidity with NCDs, or multimorbidity with NCDs and MH, but not multimorbidity with only MH conditions. Age was associated with multimorbidity in either category of NCDs, MH or NCD and $\mathrm{MH}$ together. Education was associated with a higher risk of having multimorbidity with NCDs, but lower risk of having multimorbidity with $\mathrm{MH}$, and there was no association of with education and having multimorbidity with NCDs and MH. Increasing wealth was associated with having multimorbidity with NCDs, but not with MH, nor having multimorbidity with NCDs and MH.

In multivariable analyses, having a greater number of conditions was substantially and significantly associated with a greater prevalence of frailty, worse disability, worse quality of life and slower walk speed, but not time to chair rise or grip strength, when controlling for sex, age, education level, marital status and wealth (table 3). Multimorbidity defined as two or more conditions was significantly associated with a greater prevalence of frailty, worse disability, worse quality of life and poorer physical performance expect for hand grip strength (table 4). In figure 1, the relationship between increasing age and multimorbidity with frailty (figure 1A), disability (figure 1B) and quality of life (figure 1C) is shown. Both discordant and concordant multimorbidity were associated with a greater prevalence of frailty, worse disability, worse quality of life and lower walk-speed, but not time to chair rise or grip strength (online supplementary appendix table 5). Discordant multimorbidity was also more strongly associated with a higher prevalence of frailty, higher disability score and worse quality of life than concordant multimorbidity (online supplementary appendix table 5), however, differences were nonsignificant when adjusting for number of conditions in the multivariable analysis (table 5). Compared with being multimorbid in the domain of NCDs alone, multimorbidity in the domains of $\mathrm{MH}$, or multimorbidity with $\mathrm{MH}$ and NCDs was associated with greater frailty, higher disability scores, lower quality of life, lower walk speed and lower hand grip strength (online supplementary appendix table 6). Proportions and overlap between people with NCDs, $\mathrm{MH}$ or communicable diseases are shown in figure 2 . Only $0.1 \%$ had a condition in all three disease domains.

\section{DISCUSSION}

This study has shown that in rural Burkina Faso, multimorbidity, defined as two or more conditions, is common, and present in nearly one quarter of the population over 40 years old. Multimorbidity is more common in women, among those who are older, those who have formal education, unmarried individuals and those of relatively higher economic status. Although limited by the number of conditions that we could collect, we found that discordant multimorbidity was as common as concordant multimorbidity, highlighting that patterns of multimorbidity are complex in this population. Multimorbidity was independently associated with a greater prevalence of frailty, worse disability, lower quality of life and lower walk speed. There was no significant difference between those with discordant versus concordant multimorbidity, after adjustment for number of conditions. However, multimorbidity with $\mathrm{MH}$ alone, or multimorbidity with $\mathrm{MH}$ and NCDs had worse measurements for all outcomes, except from time to chair rise, compared with multimorbidity with only NCDs.

Multimorbidity is a little-explored topic in low-resource settings ${ }^{18}$ and this is one of the first papers reporting the prevalence of multimorbidity such settings. There has been one previous study from Burkina Faso, conducted in a major city in 2010, which found an even higher prevalence of multimorbidity $(65 \%)$ than in this study. ${ }^{32}$ However, the earlier study population was older $(>60$ years of age) and data were collected on a wider range of conditions, many of which were highly prevalent (eg, osteoarthritis), and which encompassed a range of health states somewhat broader than our definition of a disease (eg, visual impairment, malnutrition). In our sample, the prevalence of multimorbidity was higher $(35.2 \%)$ among those of 60 years of age compared with younger adults, but not nearly as high as in the previous study from Burkina Faso. Thus, it is possible either that our results underestimate the prevalence of broad multimorbidity in 


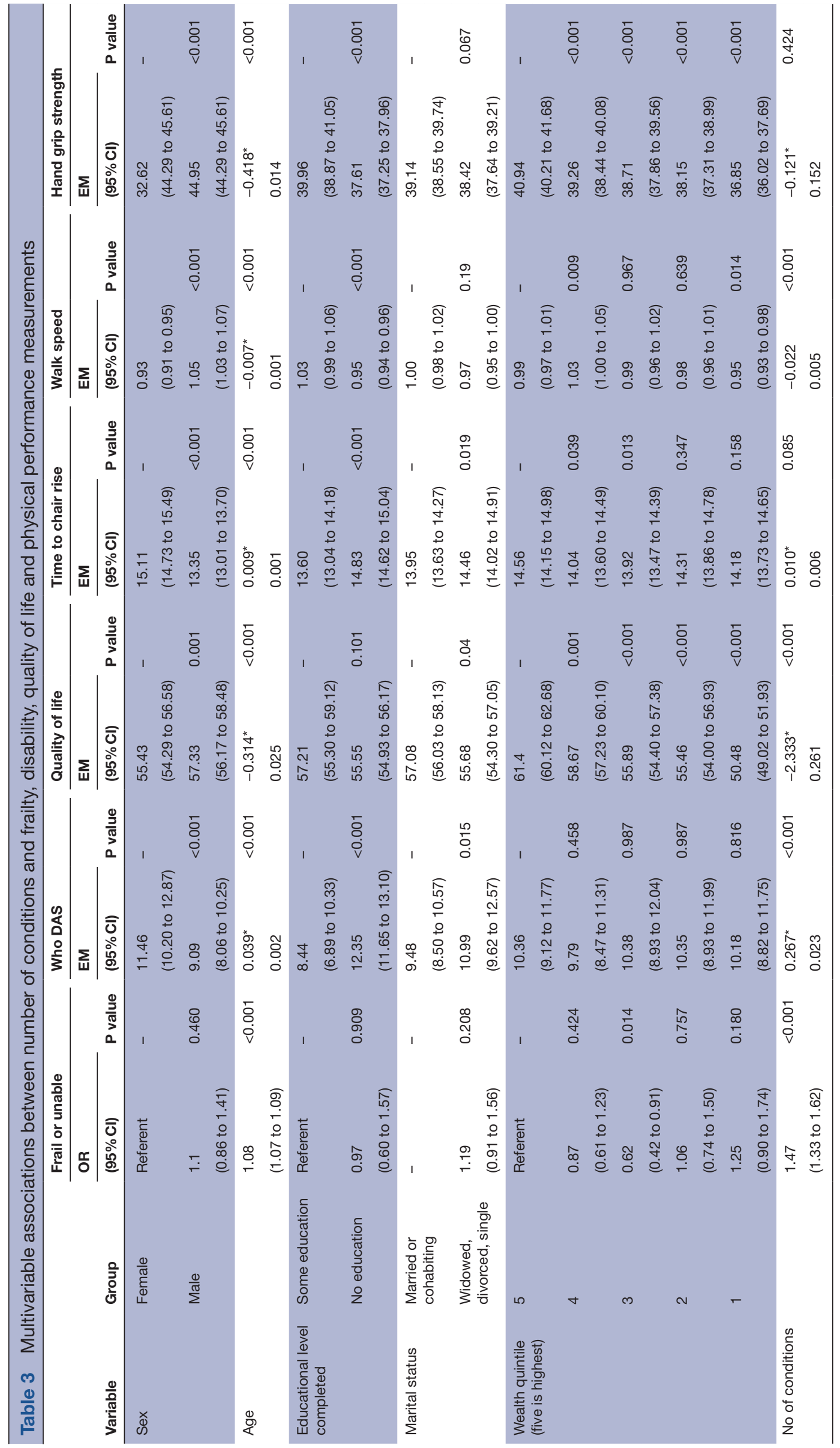




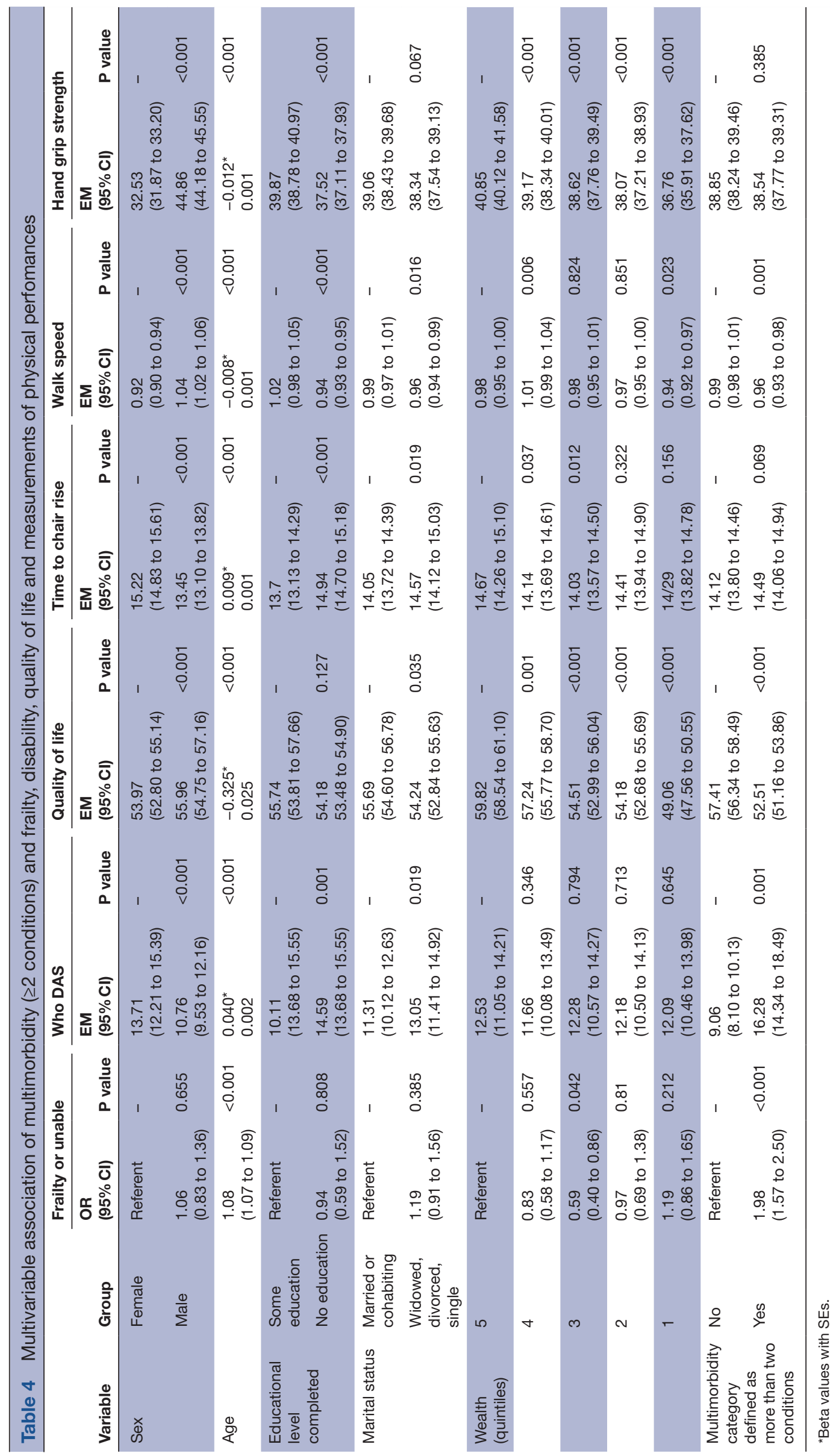



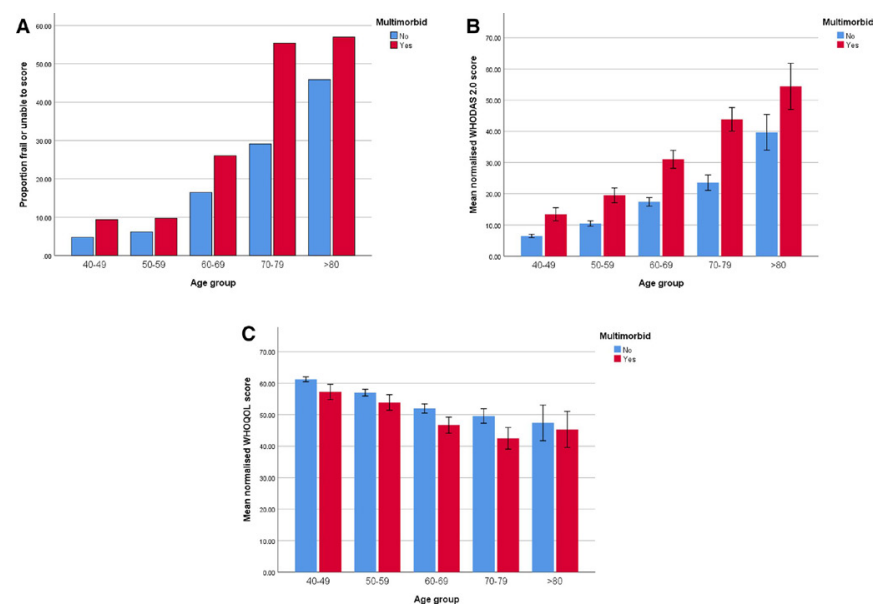

Figure 1 Frailty, disability score (WHODAS) and quality of life score (WHOQOL) according to age group and multimorbidity.

rural Burkina Faso, or that previous work overestimated the prevalence, depending on the definition of what constitutes a disease that is adopted.

We are not aware of any previous study that has looked at the patterns (or morbidity domains in which disease is occurring) of multimorbidity or ascertained its associations with frailty, disability, quality of life and physical performance in West Africa or other LICs defined as least developed. Our findings are of particular importance, because, although we cannot demonstrate causality in this cross-sectional study, it highlights that multimorbidity is at least associated with important health and well-being consequences, including limitations in physical function and decreased self-reported quality of life.

Knowledge of who suffers from multimorbidity and how morbidities are grouped can help with the design of healthcare services to treat the multimorbid patient, and support in the development of guidelines for healthcare practitioners of which other conditions to look for when a person presents with a single disease.

In contrast to findings from high-income countries, ${ }^{225152}$ our study has found that even when controlling for age, wealthier people are more likely to have multimorbidity than those who are poorer, this is especially the case for multimorbidity with NCDs. This may reflect the shifting patterns of diseases and risk factors with individual wealth seen as countries' gross domestic product improves. ${ }^{53}$ Obesity in particular affects those who are relatively wealthy in LICs and LMICs and associated cardiometabolic diseases affect the highest wealth quintile. $^{53}$ The findings may also reflect that wealthy people have better access to healthcare and are more likely to have their conditions diagnosed, ${ }^{52}{ }^{54}$ although work has shown that healthcare access is universally poor in LICs. ${ }^{556}$ While we have found that the socioeconomic associations with multimorbidity in this low-income country are different to those seen in high-income countries, we have shown that the associations in terms of functional outcomes are similar across socioeconomic strata. Thus, ultimately, the meaning of multimorbidity in terms of consequences to the patients, the healthcare system which treats them and the families that care for them, is likely to be similar no matter what income level patients come from.

We have shown that in this population, having two or more conditions in the discordant multimorbidity category is just as likely as having two or more conditions in the same domain; thus, in healthcare structures that have hitherto provided siloed care, adopting a more holistic care provision strategy could provide an avenue towards better health. The high prevalence of discordant multimorbidity highlights that such approaches cannot be limited to the management of concordant clusters of morbidities, such as diabetes, hypertension and dyslipidaemia; such an approach has failed to provide effective, joined-up care in high-income countries, ${ }^{57}$ and replicating this model in developing countries would therefore be a missed opportunity. However, because the number of conditions we investigated in this study were limited, we were unable to conduct a more nuanced cluster analysis.

Frailty, disability and reduced quality of life are strongly and independently associated with increasing number of conditions. However, these findings also indicate that discordant multimorbidity is no different to concordant multimorbidity in its association with poor individual outcomes, when controlling for number of conditions. This is in contrast with what has been found previously with patients with discordant multimorbidities typically having poorer health-related quality of life and worse clinical outcomes. ${ }^{6} 1358$ Interestingly, we found that people who have multimorbidity with only $\mathrm{MH}$ conditions or multimorbidity with $\mathrm{MH}$ conditions and NCDs have worse outcomes than those with only NCDs. The finding that people with NCDs and MH have worse outcomes than those with NCDs alone has been found in other countries, including high income countries (HICs) ${ }^{6}$ However, our findings suggest that those with multimorbidity in the domain of $\mathrm{MH}$ alone were the worse off in terms of outcomes that we assessed. A reason for this could be that people with NCDs have greater access to healthcare and have their needs addressed more thoroughly than people with $\mathrm{MH}$ conditions. ${ }^{59} \mathrm{~A}$ previous paper from South Africa found that patterns of multimorbidity affect healthcare utilisation, potentially supporting our hypothesis; ${ }^{13}$ however, this finding needs to be explored in further studies.

It is not possible from this cross-sectional analysis to know if the relationship between increasing numbers of morbidities and frailty, disability, low quality of life and poor physical performance is causal. However, given life expectancy in Burkina Faso increased from 49.5 in 1990 to 60.8 in $2017,{ }^{31}$ knowledge of high prevalence of multimorbidity and these associations is cause for concern in a growing population with few healthcare resources.

Hence, preventative and management interventions needs to be done in order to ensure that the health 


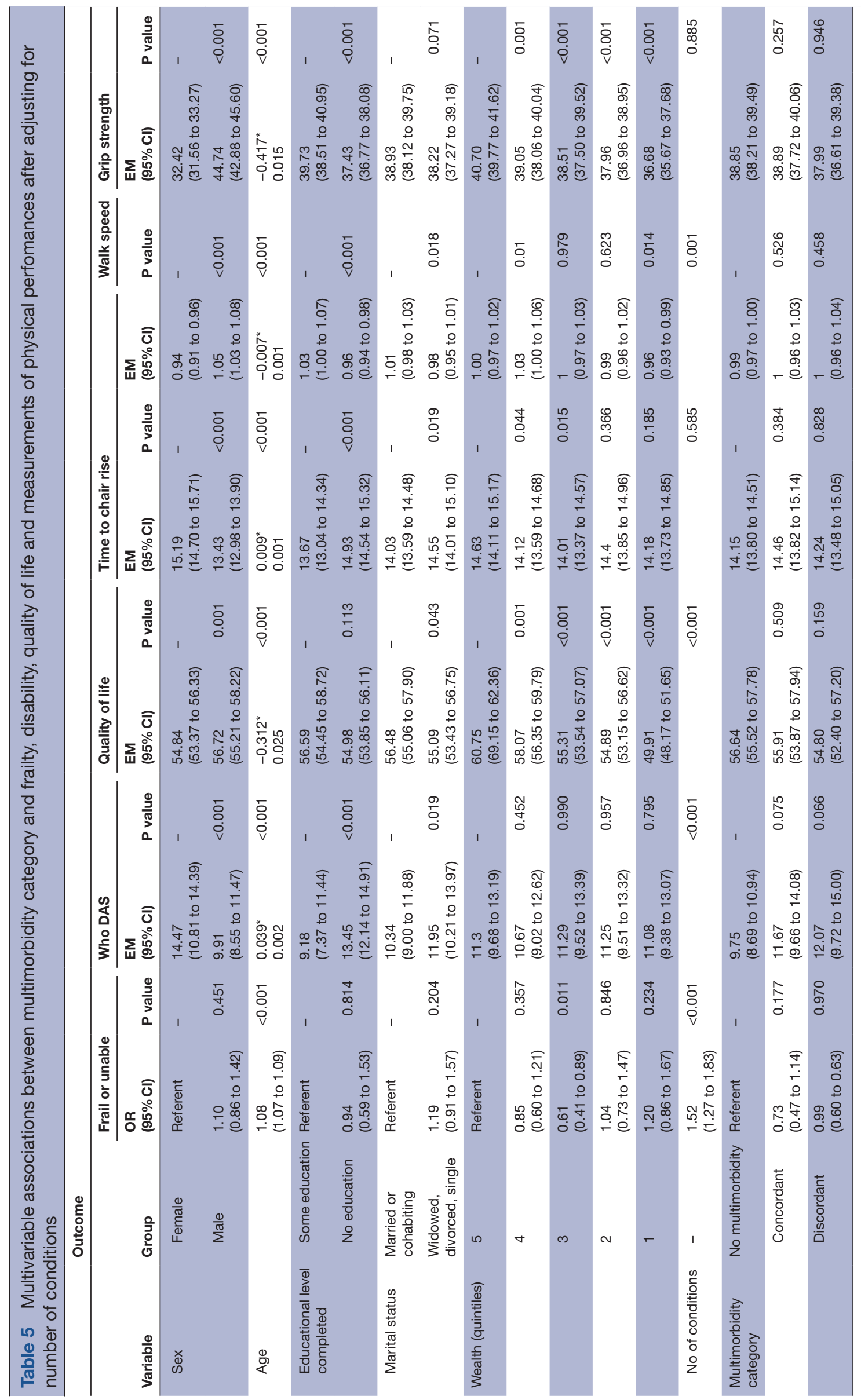




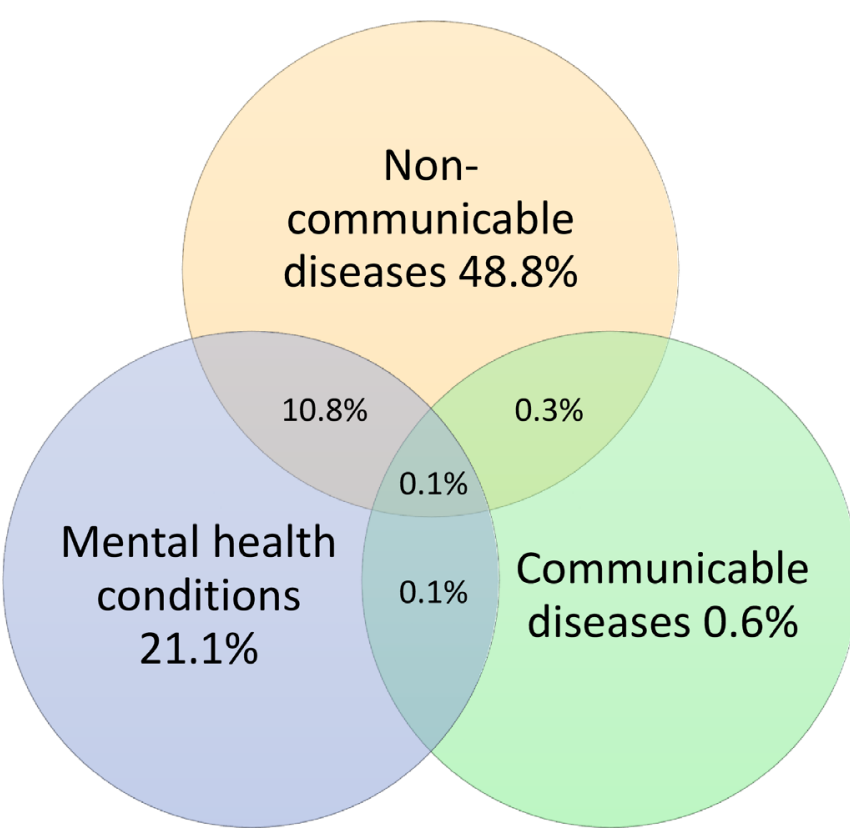

Figure 2 Proportions and overlap between people with non-communicable diseases, mental health conditions and communicable diseases.

system can deal with this increasing burden of disease and its downstream consequences.

The mismatch between prevalence of multimorbidity and research output in LMICs (only 5\% of multimorbidity research originates from LMICs) is well known. ${ }^{618}$ This study contributes to filling that knowledge gap, yet further research on the clusters of multimorbidity, their associations with adverse outcomes and health system solutions to prevent and manage multimorbidity is urgently required. Data collection that extends beyond a priori defined morbidities commonly seen in highincomes settings, to include locally determined and prioritised conditions must increasingly drive this area of research.

This study has several limitations. Even though multimorbidity prevalence was high, the number of conditions evaluated was limited, and it is likely that our multimorbidity prevalence estimates are conservative. Moreover, many of the NCDs (cancer, heart and respiratory disease) and HIV in our study relied on self-report of a diagnosis from a medical professional. However, many people in this population lack the financial resources or awareness to seek medical care for their conditions, further underestimating true prevalence and perhaps overestimating multimorbidity prevalence in people in the high income category. There is especially likely to be under-reporting of HIV in our study, due to the stigma associated with the condition. While we relied mostly on self-reported diagnoses in this study, restricting our analysis to objective measurements did not substantially change our results (online supplementary appendix table 3 ). We were not able to capture information on all chronic conditions, for example, osteoarthritis or visual impairment, in our survey, meaning that our estimates should be considered a lower bound on the true prevalence of multimorbidity in this population. However, we intentionally captured as broad spectrum of conditions as possible, and reported on 11 common conditions, similar to that seen in other studies in similar settings. ${ }^{12}$

This study is also cross-sectional, limiting our ability to assess causation. Nonetheless, longitudinal studies in high-income countries have pointed to a causal relationship between multimorbidity and outcomes similar to those assessed here. ${ }^{6061}$ Finally, participants were selected only from one area of Burkina Faso and hence our findings may not generalise nationally or internationally.

\section{CONCLUSIONS}

Burkina Faso is one of the poorest countries in the world, yet these findings show that chronic multimorbidity is already highly prevalent and associated with adverse health conditions. This research adds to a growing body of literature that highlights multimorbidity as a global health issue of major significance. Investment is needed by researchers, development agencies and national governments to prioritise understanding of multimorbidity in lower income settings, and to develop and test interventions to prevent multimorbidity and mitigate their adverse consequences.

\section{Author affiliations}

${ }^{1}$ Institute of Applied Health Research, University of Birmingham, Birmingham, UK ${ }^{2}$ School of Demography, The Australian National University, Canberra, Australian Capital Territory, Australia

${ }^{3}$ AGE Research Group, NIHR Newcastle Biomedical Research Centre, Newcastle University, Newcastle upon Tyne, UK

${ }^{4}$ Newcastle Upon Tyne Hospitals NHS Foundation Trust, Newcastle upon Tyne, UK ${ }^{5}$ Massachusetts General Hospital, Boston, Massachusetts, USA

${ }^{6}$ Africa Health Research Institute, KwaZulu-Natal, South Africa

${ }^{7}$ Heidelberg Institute of Global Health (HIGH), Faculty of Medicine and University Hospitals, University of Heidelberg, Heidelberg, Germany

${ }^{8}$ Centre de Recherche en Sante de Nouna, Nouna, Burkina Faso

${ }^{9}$ Division of Primary Care and Population Health, Department of Medicine, Stanford University, Stanford, California, USA

${ }^{10}$ Institute for Global Health, University College London, London, UK

${ }^{11}$ MRC/Wits Rural Public Health \& Health Transitions Research Unit (Agincourt), University of the Witwatersrand, Johannesburg-Braamfontein, South Africa

${ }^{12}$ Department of Epidemiology \& Harvard Center for Population and Development Studies, Harvard T.H. Chan School of Public Health, Boston, MA, USA

${ }^{13}$ Division of Infectious Diseases, Massachusetts General Hospital, Harvard Medical School, Boston, Massachusetts, USA

Twitter Guy Harling @harlingg and Justine I Davies @drjackoids

Contributors TB and GH conceived and designed the overall CSRN CHAS study. $\mathrm{MB}, \mathrm{GH}, \mathrm{LO}$ and AS co-ordinated baseline data collection and preparation. JID, $\mathrm{CP}, \mathrm{JM}, \mathrm{PG}, \mathrm{MS}$ and MDW contributed to the design of the CSRN CHAS household survey. JID, CP, MS and MDW designed the current study. MLO conducted the analysis, wrote and revised the manuscript. JID supervised the analysis, write up and development of the manuscript. All authors substantively reviewed manuscripts, inputted into revisions and approved the final manuscript.

Funding Support for the CRSN Heidelberg Aging Study and for TB was provided by the Alexander von Humboldt Foundation through the Alexander von Humboldt Professor award to Till Bärnighausen, funded by the German Federal Ministry of Education and Research. CFP is supported by the ANU Futures Scheme. Professor Witham acknowledges support from the NIHR Newcastle Biomedical Research Centre. MJS receives research support from the National Institutes of Health (R01 HL141053 and R01 AG 059504 and P30Al060354). GH is supported by a fellowship 
from the Wellcome Trust and Royal Society 210479/Z/18/Z. JMG was supported by Grant Number T32 Al007433 from the National Institute of Allergy and Infectious Diseases.

\section{Competing interests None declared.}

Patient and public involvement Patients and/or the public were not involved in the design, or conduct, or reporting or dissemination plans of this research.

Patient consent for publication Not required.

Ethics approval Ethical approval was obtained from Ethics Commission I of the medical faculty Heidelberg (S-120/2018), the Burkina Faso Comité d'Ethique pour la Recherche en Santé (CERS) in Ouagadougou (2018-4-045) and the Institutional Ethics Committee (CIE) of the CRSN (2018-04). Oral assent was sought from all village elders. Written informed consent was obtained from each participant and a literate witness assisted in cases of illiteracy. All participants with test results indicating hypertension, diabetes, dyslipidaemia or anaemia were contacted and provided with referral to the appropriate level of care.

Provenance and peer review Not commissioned; externally peer reviewed.

Data availability statement Data may be obtained from a third party and are not publicly available. Data are not publicly available as consent was not given by participants for this to take place. This is in part because entire age cohorts of some villages are included in the data set, potentially allowing for deductive disclosure with sufficient local information. For this reason, anonymised data are available from CHAS study data controllers only following signature of a data use agreement restricting onward transmission. Anyone wishing to replicate the analyses presented, or conduct further collaborative analyses using CHAS (which are welcomed and considered based on a letter of intent), should contact Dr Guy Harling (g.harling@ucl.ac.uk) in the first instance.

Open access This is an open access article distributed in accordance with the Creative Commons Attribution Non Commercial (CC BY-NC 4.0) license, which permits others to distribute, remix, adapt, build upon this work non-commercially, and license their derivative works on different terms, provided the original work is properly cited, appropriate credit is given, any changes made indicated, and the use is non-commercial. See: http://creativecommons.org/licenses/by-nc/4.0/.

\section{ORCID iDs}

Maria Lisa Odland http://orcid.org/0000-0003-4340-7145

Guy Harling http://orcid.org/0000-0001-6604-491X

\section{REFERENCES}

1 Alkema L, Chou D, Hogan D, et al. Global, regional, and national levels and trends in maternal mortality between 1990 and 2015, with scenario-based projections to 2030: a systematic analysis by the un maternal mortality estimation Inter-Agency group. Lancet 2016;387:462-74.

2 World Health Organization. Global health Observatory data, 2019. Available: https://www.who.int/gho/hiv/epidemic_status/deaths_text/ en/

3 World Health Organization. Children: reducing mortality 2019. Available: https://www.who.int/newsroom/fact-sheets/detail/ children-reducing-mortality

4 Aboderin IAG, Beard JR. Older people's health in sub-Saharan Africa. Lancet 2015;385:e9-11.

5 Bor J, Herbst AJ, Newell M-L, et al. Increases in adult life expectancy in rural South Africa: valuing the scale-up of HIV treatment. Science 2013;339:961-5.

6 The Academy of Medical Sciences. Multimorbidity: a priority for global health research, 2018.

7 Patel V, Saxena S, Lund C, et al. The Lancet Commission on global mental health and sustainable development. Lancet 2018;392:1553-98.

8 Islam SMS, Purnat TD, Phuong NTA, et al. Non-Communicable diseases (NCDS) in developing countries: a symposium report. Global Health 2014;10:81.

9 United nations. Sustainable development goals, 2019. Available: https://sustainabledevelopment.un.org/sdgs

10 Mendenhall E, Kohrt BA, Norris SA, et al. Non-Communicable disease syndemics: poverty, depression, and diabetes among lowincome populations. Lancet 2017;389:951-63.

11 Gomez-Olive FX, Schröders J, Aboderin I, et al. Variations in disability and quality of life with age and sex between eight lower income and middle-income countries: data from the indepth WHOSAGE collaboration. BMJ Glob Health 2017;2:e000508.
12 Chang AY, Gómez-Olivé FX, Payne C, et al. Chronic multimorbidity among older adults in rural South Africa. BMJ Glob Health 2019;4:e001386

13 Chang AY, Gómez-Olivé FX, Manne-Goehler J, et al. Multimorbidity and care for hypertension, diabetes and HIV among older adults in rural South Africa. Bull World Health Organ 2019;97:10-23.

14 Agyepong IA, Sewankambo N, Binagwaho A, et al. The path to longer and healthier lives for all Africans by 2030: the Lancet Commission on the future of health in sub-Saharan Africa. Lancet 2018;390:280359.

15 Fortin M, Lapointe L, Hudon C, et al. Multimorbidity and quality of life in primary care: a systematic review. Health Qual Life Outcomes 2004;2:51.

16 Wolff JL, Starfield B, Anderson G, Prevalence AG. Prevalence, expenditures, and complications of multiple chronic conditions in the elderly. Arch Intern Med 2002;162:2269-76.

17 Cortaredona S, Ventelou B. The extra cost of comorbidity: multiple illnesses and the economic burden of non-communicable diseases. BMC Med 2017;15:216

$18 \mathrm{Xu} \mathrm{X,} \mathrm{Mishra} \mathrm{GD,} \mathrm{Jones} \mathrm{M.} \mathrm{Mapping} \mathrm{the} \mathrm{global} \mathrm{research} \mathrm{landscape}$ and knowledge gaps on multimorbidity: a bibliometric study. J Glob Health 2017;7:010414.

19 Park B, Ock M, Lee HA, et al. Multimorbidity and health-related quality of life in Koreans aged 50 or older using KNHANES 20132014. Health Qual Life Outcomes 2018;16:186.

20 Garin N, Koyanagi A, Chatterji S, et al. Global multimorbidity patterns: a cross-sectional, population-based, Multi-Country study. $J$ Gerontol A Biol Sci Med Sci 2016;71:205-14.

21 Fabbri E, An Y, Zoli M, et al. Association between accelerated multimorbidity and age-related cognitive decline in older Baltimore longitudinal study of aging participants without dementia. J Am Geriatr Soc 2016;64:965-72.

22 Barnett K, Mercer SW, Norbury M, et al. Epidemiology of multimorbidity and implications for health care, research, and medical education: a cross-sectional study. Lancet 2012;380:37-43.

23 Ahmadi B, Alimohammadian M, Yaseri M, et al. Multimorbidity: epidemiology and risk factors in the Golestan cohort study, Iran: a cross-sectional analysis. Medicine 2016;95:e2756

24 Aarts S, van den Akker M, Tan FES, et al. Influence of multimorbidity on cognition in a normal aging population: a 12-year follow-up in the Maastricht aging study. Int J Geriatr Psychiatry 2011;26:1046-53.

25 Kieny M-P, Evans DB, Schmets G, et al. Health-system resilience: reflections on the Ebola crisis in Western Africa. Bull World Health Organ 2014;92:850.

26 Wister A, Lear S, Schuurman N, et al. Development and validation of a multi-domain multimorbidity resilience index for an older population: results from the baseline Canadian longitudinal study on aging. BMC Geriatr 2018;18:170.

27 Schiltz NK, Warner DF, Sun J, et al. Identifying specific combinations of multimorbidity that contribute to health care resource utilization: an analytic approach. Med Care 2017;55:276-84.

28 Ng SK, Tawiah R, Sawyer M, et al. Patterns of multimorbid health conditions: a systematic review of analytical methods and comparison analysis. Int J Epidemiol 2018;47:1687-704.

29 World Health Organization. Multimorbidity. Geneva, Switzerland: World Health Organization, 2016.

30 World Bank. The world bank in Burkina Faso, 2019. Available: http:// www.worldbank.org/en/country/burkinafaso/overview

31 United Nations Development Programme. Burkina Faso human development indicators 2018. Available: http://hdr.undp.org/en/ countries/profiles/BFA [Accessed 25 Jun 2019].

32 Hien H, Berthé A, Drabo MK, et al. Prevalence and patterns of multimorbidity among the elderly in Burkina Faso: cross-sectional study. Trop Med Int Health 2014;19:1328-33.

33 Sié A, Louis VR, Gbangou A, et al. The health and demographic surveillance system (HDSS) in Nouna, Burkina Faso, 1993-2007. Glob Health Action 2010;3. doi:10.3402/gha.v3i0.5284. [Epub ahead of print: 14 Sep 2010]

34 Hartung C. Open data kit: tools to build information services for developing regions, 2010

35 Hall KS, Hendrie HC, Brittain HM, et al. The development of a dementia screening interview in 2 distinct languages. Int $J$ Method Psych 1993;3:1-28.

36 Plummer F, Manea L, Trepel D, et al. Screening for anxiety disorders with the GAD-7 and GAD-2: a systematic review and diagnostic metaanalysis. Gen Hosp Psychiatry 2016;39:24-31.

37 Spitzer RL, Kroenke K, Williams JB. Validation and utility of a selfreport version of PRIME-MD: the $\mathrm{PHQ}$ primary care study. primary care evaluation of mental disorders. patient health questionnaire. JAMA 1999;282:1737-44. 
38 Kroenke K, Spitzer RL, Williams JB. The PHQ-9: validity of a brief depression severity measure. J Gen Intern Med 2001;16:606-13.

39 Fried LP, Tangen CM, Walston J, et al. Frailty in older adults: evidence for a phenotype. J Gerontol A Biol Sci Med Sci 2001;56:M146-57.

40 Witham MD, Davies JI, Bärnighausen T, et al. Frailty and physical performance in the context of extreme poverty: a population-based study of older adults in rural Burkina Faso. Wellcome Open Res 2019;4:135.

41 Guralnik JM, Simonsick EM, Ferrucci L, et al. A short physical performance battery assessing lower extremity function: association with self-reported disability and prediction of mortality and nursing home admission. J Gerontol 1994;49:M85-94.

42 Roberts HC, Denison HJ, Martin HJ, et al. A review of the measurement of grip strength in clinical and epidemiological studies: towards a standardised approach. Age Ageing 2011;40:423-9.

43 Filmer D, Pritchett LH. Estimating wealth effects without expenditure data--or tears: an application to educational enrollments in states of India. Demography 2001;38:115-32.

44 Hall KS, Gao S, Emsley CL, et al. Community screening interview for dementia (CSI 'D'); performance in five disparate study sites. Int $J$ Geriatr Psychiatry 2000;15:521-31.

45 Pritchard JM, Kennedy CC, Karampatos S, et al. Measuring frailty in clinical practice: a comparison of physical frailty assessment methods in a geriatric out-patient clinic. BMC Geriatr 2017;17:264.

46 Payne CF, Wade A, Kabudula CW, et al. Prevalence and correlates of frailty in an older rural African population: findings from the HAALSI cohort study. BMC Geriatr 2017;17:293.

47 Radloff L. The CES-D scale: a self-report depression scale for research in the general population. Appl Psychol Measurement 1977:1:385-401.

48 Garin O, Ayuso-Mateos JL, Almansa J, et al. Validation of the "World Health Organization Disability Assessment Schedule, WHODAS-2" in patients with chronic diseases. Health Qual Life Outcomes 2010;8:51.

49 Schmidt S, Mühlan H, Power M. The EUROHIS-QOL 8-item index: psychometric results of a cross-cultural field study. Eur $\mathrm{J}$ Public Health 2006;16:420-8.

50 Snoek FJ, Bremmer MA, Hermanns N. Constructs of depression and distress in diabetes: time for an appraisal. Lancet Diabetes Endocrinol 2015;3:450-60.
51 Singer L, Green M, Rowe F, et al. Trends in multimorbidity, complex multimorbidity and multiple functional limitations in the ageing population of England, 2002-2015. J Comorb 2019:9:2235042X1987203.

52 Rosengren A, Smyth A, Rangarajan S, et al. Socioeconomic status and risk of cardiovascular disease in 20 low-income, middleincome, and high-income countries: the prospective urban rura epidemiologic (pure) study. Lancet Glob Health 2019;7:e748-60.

53 Templin T, Cravo Oliveira Hashiguchi T, Thomson B, et al. The overweight and obesity transition from the wealthy to the poor in low- and middle-income countries: A survey of household data from 103 countries. PLoS Med 2019;16:e1002968.

54 Palafox B, McKee M, Balabanova D, et al. Wealth and cardiovascular health: a cross-sectional study of wealth-related inequalities in the awareness, treatment and control of hypertension in high-, middle- and low-income countries. Int $J$ Equity Health 2016;15:199.

55 Geldsetzer P, Manne-Goehler J, Marcus M-E, et al. The state of hypertension care in 44 low-income and middle-income countries: a cross-sectional study of nationally representative individual-level data from 1.1 million adults. Lancet 2019;394:652-62.

56 Manne-Goehler J, Geldsetzer P, Agoudavi K, et al. Health system performance for people with diabetes in 28 low- and middle-income countries: a cross-sectional study of nationally representative surveys. PLoS Med 2019;16:e1002751.

57 Whitty CJ. Harveian oration 2017: triumphs and challenges in a world shaped by medicine. Clin Med 2017;17:537-44.

58 Prados-Torres A, Calderón-Larrañaga A, Hancco-Saavedra J, et al. Multimorbidity patterns: a systematic review. J Clin Epidemiol 2014;67:254-66.

59 Demyttenaere K, Bruffaerts R, Posada-Villa J, et al. Prevalence, severity, and unmet need for treatment of mental disorders in the world Health organization world mental health surveys. JAMA 2004:291:258190.

60 Rivera-Almaraz A, Manrique-Espinoza B, Ávila-Funes JA, et al. Disability, quality of life and all-cause mortality in older Mexican adults: association with multimorbidity and frailty. BMC Geriatr 2018;18:236.

61 Vetrano DL, Palmer K, Marengoni A, et al. Frailty and multimorbidity: a systematic review and meta-analysis. J Gerontol A Biol Sci Med Sci 2019;74:659-66. 
Correction: Epidemiology of multimorbidity in conditions of extreme poverty: a population-based study of older adults in rural Burkina Faso

Odland ML, Payne C, Witham MD, et al. Epidemiology of multimorbidity in conditions of extreme poverty: a population-based study of older adults in rural Burkina Faso. BMJ Global Health 2020;5: e002096.

In the published version, part of the sampling scheme was misrepresented due to a confusion over versions of the study protocol. The original text read:

In all villages with fewer than 90 adults aged over 40, all adults were selected to take part.

This should in fact have read:

In all villages $(n=6)$ with fewer than 50 adults aged over 40 , all adults were selected to take part.

Open access This is an open access article distributed in accordance with the Creative Commons Attribution Non Commercial (CC BY-NC 4.0) license, which permits others to distribute, remix, adapt, build upon this work non-commercially, and license their derivative works on different terms, provided the original work is properly cited and the use is noncommercial. See: http://creativecommons.org/licenses/by-nc/4.0/.

C BMJ Publishing Group Limited 2020. Re-use permitted under CC BY-NC. No commercial re-use. See rights and permissions. Published by BMJ.

BMJ Global Health 2020;5:e002096corr1. doi:10.1136/bmjgh-2019-002096corr1

Check for updates 